\title{
Three theories for reconciling the linearity of egocentric distance perception with distortion of shape on the ground plane
}

\author{
Jack M. Loomis \\ University of California, Santa Barbara, CA, USA
}

\begin{abstract}
The focus here is on the paradoxical finding that whereas visually perceived egocentric distance is proportional to physical distance out to at least $20 \mathrm{~m}$ under full-cue viewing, there are large distortions of shape within the same range, reflecting a large anisotropy of depth and frontal extents on the ground plane. Three theories of visual space perception are presented, theories that are relevant to understanding this paradoxical result. The theory by Foley, Ribeiro-Filho, and Da Silva is based on the idea that when the visual system computes the length of a visible extent, the effective visual angle is a non-linear increasing function of the actual visual angle. The theory of Durgin and Li is based on the idea that two angular measures, optical slant and angular declination, are over-perceived. The theory of Ooi and He is based on both a default perceptual representation of the ground surface in the absence of visual cues and the "sequential surface integration process" whereby an internal representation of the visible ground surface is constructed starting from beneath the observer's feet and extending outward. Keywords: visual space perception, vision, depth perception.
\end{abstract}

Received 07 December 2013; received in revised form 31 March 2014; accepted 31 March 2014. Available online 25 November 2014.

\section{Introduction}

The topic of visual space perception is unique because of the huge gulf between how it is viewed by novices and how it is viewed by those researchers who have delved into the topic. On the one hand, because the experimental methods need not be technical, anyone can begin doing research by placing some visual targets on the ground and asking participants to make estimates of distance. On the other, research on visual space perception over the many decades has led to both an appreciation of its subtlety and a theoretical understanding that defies common sense and goes well beyond the typical textbook treatment (e.g., Foley, Ribeiro-Filho, \& Da Silva, 2004; Gogel, 1990; Howard \& Rogers, 2012; Li \& Durgin, 2010; Loomis, Da Silva, Fujita, \& Fukusima, 1992; Sedgwick, 1986; B. Wu, Ooi, \& He, 2004; Yang \& Purves, 2003; Zhou, He, $\&$ Ooi, 2013).

Illustrating the subtlety of visual space perception is the paradoxical finding that whereas visually perceived egocentric distance under full cue viewing

Jack M. Loomis, Department of Psychological and Brain Sciences, University of California, Santa Barbara, Santa Barbara, CA 93106, USA. Correspondence regarding this article should be addressed to: Jack M. Loomis, Department of Psychological and Brain Sciences, University of California, Santa Barbara, Santa Barbara, CA 93106, USA. E-mail: jmloomis99@gmail.com is a linear function of physical distance out to at least $20 \mathrm{~m}$, there are large distortions of exocentric distance and shape within the same range, reflecting a large anisotropy of depth and frontal extents on the ground plane. This paradoxical finding was first pointed out by Loomis et al. (1992), although prior research had already indicated that egocentric distance perception and exocentric distance perception rely on different cues and different processes (Baird \& Biersdorf, 1967; Foley, 1980; Gogel, 1977).

To elaborate on this difference between egocentric distance perception and exocentric distance perception under full cue viewing, we first note that a large number of studies show that the perceived distance of targets presented on the ground plane under full-cue viewing is a linear or nearly linear function (with zero intercept) of target distance out to at least $20 \mathrm{~m}$. This is true whether verbal report, blind walking, or a triangulation method is used to provide an indicator of perceived distance (see summaries in Loomis \& Philbeck, 2008). Whereas action-based estimates are accurate (linear functions with slopes near 1.0), verbal reports typically show underestimation (slopes near 0.7-0.8). Thus, regardless of which task is more valid, perceived egocentric distance is proportional to physical distance. Because visual direction to a single target point is generally thought to be accurate (e.g., Gogel, 1982; 1990, Ooi, B. Wu, \& He, 2001; Zhou et al., 2013; for contrary 
evidence, see Li, Phillips, \& Durgin, 2011), the finding of linear egocentric distance perception would imply that the perceived locations of visual targets from some fixed viewpoint are accurate or, if the slope is less than unity, uniformly pulled toward the viewpoint. Yet, under the same conditions of full-cue viewing of targets, numerous studies have shown large distortions of perceived shape or perceived relative exocentric distance, when the shapes or exocentric distances are specified by targets on the ground plane. Two types of perceptual distortion have received considerable attention. One is the distortion of shape accompanying a configuration consisting of frontal extents and depth extents on the group plane, as shown in Figure 1. The two panels show a configuration of three balls lying on the ground within a distance of $10 \mathrm{~m}$. In the left panel, the distance between the two left balls is about double that between the two balls in front. The right panel shows the same configuration from an orthogonal perspective. The change in apparent shape of the configuration with the shift in viewpoint is readily apparent even for an observer viewing the real configuration under full-cue cues. Many studies have reported that depth extents on the ground plane are perceived to be smaller, sometimes more than $50 \%$ smaller, than physically equal frontal extents (Beusmanns, 1998; Bian \& Andersen, 2011; Foley, 1968, 1972; Kudoh, 2005; Levin \& Haber, 1993; Li \& Durgin, 2010, 2013; Loomis et al., 1992; Loomis \& Philbeck, 1999; Loomis, Philbeck, \& Zahorik, 2002; Norman, Todd, Perotti, \& Tittle, 1996; Toye, 1986; Wagner, 1985; B. Wu et al., 2004). This distortion of shape is not only evident in simple configurations like that in Figure 1 but also in complex patterns, like those composed of sidewalk tiles as in Figure 2. The other type of distortion is not of shape per se but of misperceiving the relative sizes of two or more depth extents. When observers instruct an assistant to mark

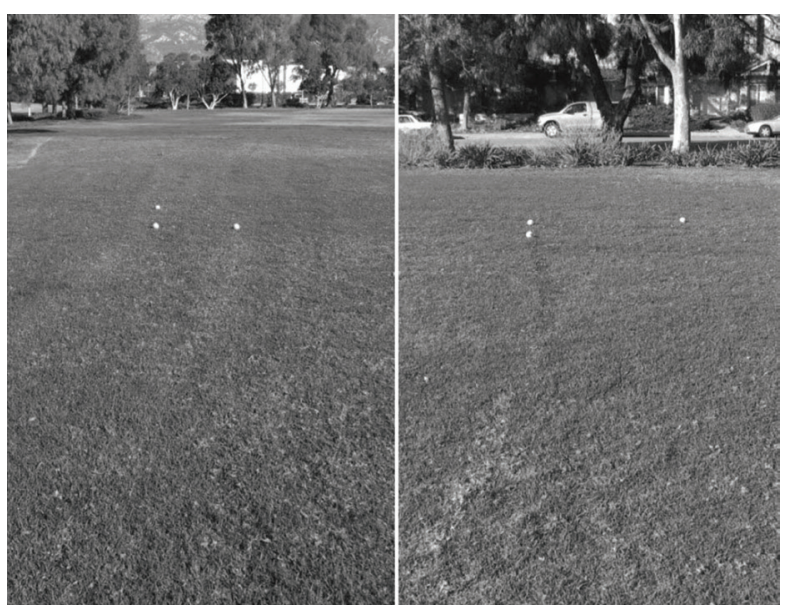

Figure 1. Two photos of the same configuration of three balls from orthogonal perspectives. In the left panel, the separation of the two balls on the left was about twice that of the two balls at the front. The perceived configuration appears much different from the physical configuration even when viewing the actual scene with binocular vision.

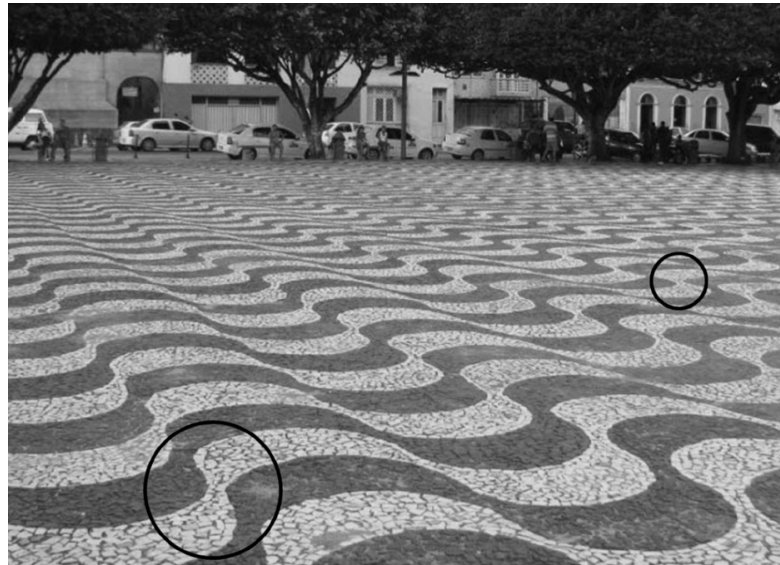

Figure 2. Photograph of a plaza in Manaus, Brazil. Even though the sidewalk tiles have a uniform pattern over the region shown, the perceived shapes of sections of the tiles vary with position (see two circles).

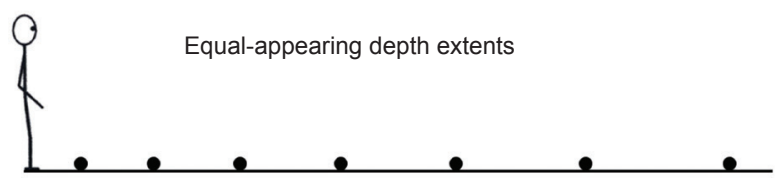

Figure 3. Side-view depiction of equal-appearing physical extents varying in distance from an observer (e.g., Gilinsky, 1951). Observers need to demarcate larger and larger physical extents to appear equal in size as the extents are farther and farther away.

out equal-appearing intervals on the ground on a line extending directly from the observer, the markers define successive depth intervals of increasing extent, as illustrated in Figure 3 (Gilinsky, 1951; Ooi \& He, 2007). Similarly, if observers are asked to adjust a distant depth interval to appear equal to a much closer depth interval, the more distant interval needs to be significantly large than the nearer interval (Beusmanns, 1998). The focus in this article will be on theories for explaining the shape distortion of frontal and depth extents under conditions where perceived egocentric distance is proportional to physical distance.

\section{Hypothesis of dissociation of egocentric and exocentric processing}

In the 1990s, the author and his colleagues hypothesized that the inconsistency between the linearity of perceived egocentric distance and the distortion of shape is a result of some dissociation within the visual system--that the perceptual representation of locations in 3-D space on the ground plan does not constrain the representation of shape, with the two types of representation reflecting different types of processing (Loomis et al., 1992; Loomis, Da Silva, Philbeck, \& Fukusima, 1996). Since then, three quantitative theories have been proposed that go a long way in elucidating whether such a dissociation is likely and, if so, how it might arise. 


\section{Three theories}

Theory of Foley, Ribeiro-Filho, and Da Silva. This theory originated with earlier experimental work by Foley and others (Foley, 1968, 1991; Higashiyama \& Ueyama, 1988), which showed that frontal extents are perceived to be systematically larger than equal depth extents (in particular, egocentric depth extents). Foley (1991) proposed a model of visual geometry with an associated metric to account for these distortions in perceived extent, in which, for a given extent, its effective visual angle is larger than the actual visual angle. Moreover, effective visual angle is a non-linear increasing function of the actual visual angle. Because the effective visual angle is used by the visual system in the computation of perceived extent, the perceived extent differs from the physical extent. Foley et al. (2004) reported an experiment and a theory to account for the data from the experiment. In the experiment, labeled vertical targets were placed in a large outdoor field and observers verbally estimated the distances between all pairs of the targets as well as the egocentric distances to each target, with both types of judgments performed under monocular and binocular viewing (which differed only slightly). The authors fit a formal quantitative model to the data that was a slight variant of the 1991 theory. The model includes a function for perceived egocentric distance, which is slightly nonlinear for binocular viewing but essentially linear for monocular viewing. The model fits the full set of data, egocentric and exocentric, much better than a more general model, which is a generalization of the classical size-distance invariance. A virtue of the model is that it also applies to configurations of targets viewed binocularly in the absence of a visible ground surface. Because the formal model simultaneously accounts for linear (or nearly linear) egocentric distance perception and distortion of exocentric shape, it entails a unified visual representation of space.

Loomis et al. (2002, p. 1210) speculated that when the depth and frontal extents are adjacent on the ground plane, the judgment of shape (relative extent) leads to a greater distortion than that which is predicted from judgments of depth and width extents presented in isolation. Interestingly, although the Foley et al. (2004) quantitative model was based on verbal estimates of the single extents presented one at a time on the ground, the model is surprisingly accurate in predicting the results of Loomis et al. (1992; Experiments 1 and 2), in which observers adjusted the length of a depth extent on the ground (via instructions to an assistant) to perceptually match a given frontal extent that was adjacent. The left panel of Figure 4 shows the mean values of two experiments (Experiments 1 and 2 with 10 observers each) as a function of the egocentric distance to the frontal extent and the length of the frontal extent; very similar values were reported in a similar experiment by Kudoh (2005). The right panel of Figure 4 gives the predicted values of the Foley et al. (2004) model using the same model parameters used to fit the data of their experiment.

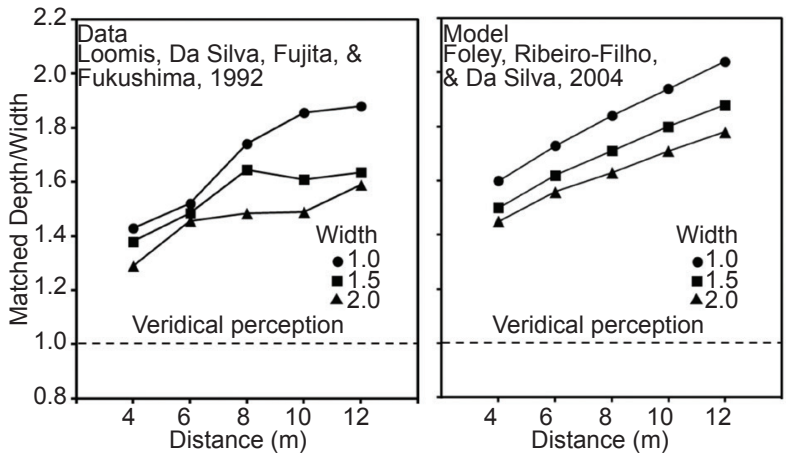

Figure 4. Left panel. Data of Experiment 2 from Loomis et al. (1992). Observers instructed an assistant to move targets defining a depth interval on the ground so that the depth interval appeared equal to a frontal interval also on the ground. The ordinate gives the ratio of the depths and widths (of the frontal intervals) that appear equal. The abscissa is the egocentric distance of the frontal interval, and the parameter is the width of the frontal interval. Right panel. Predictions of the results of the experiment from the model of Foley et al., 2004 (personal communication from John Foley, October 2013). The left panel is an adaptation of Figure 5a from Loomis, J. M., Da Silva, J.A., Fujita, N., \& Fukusima, S. S. (1992). Visual space perception and visually directed action. Journal of Experimental Psychology: Human Perception and Performance, 18, 906-921.

Not only does the model predict the increase in shape distortion with egocentric distance, it also predicts the ordering of the effect of the length of the frontal extent. However, it should be noted that Kudoh (2005) found an opposite ordering when the width extent was adjusted to perceptually match a constant depth extent used as the standard. Kudoh (2005) speculated that this might have resulted from the much smaller angular sizes of the depth extents when used as the standard in the matching procedure. It is possible that contextual range effects are involved in the matching paradigm. Even so, the success of the Foley et al. (2004) model in predicting the trends in the data of Figure 4 is striking.

Theory of Durgin and $L i$. This theory emerged from the authors' interest in the dramatic overestimation of the slopes of sloped surfaces, such as hills, whether viewed from below looking up or from above looking down (Li \& Durgin, 2009; Durgin, Li, \& Hajnal, A. (2010; Proffitt, Bhalla, Gossweiler, \& Midgett, 1995). They extended their explanation of the overestimation of slopes to encompass the misperception of visible extents on the ground plane, which is the concern here.

Figure 5 depicts two essential features of their general theory (Durgin \& Li, 2011): the overestimation of angular declination $^{1}(\alpha)$, the angle between the physical target and the eye-level plane, and the overestimation of optical slant, the angle ( $\beta$ ) between the line of sight to a patch of

"Angular declination" is also variously referred to as "gaze declination","height in the field", and "slope of regard". 


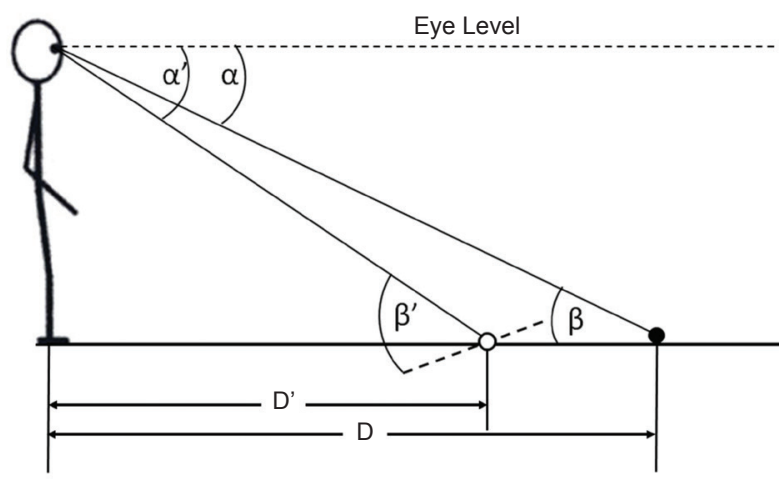

Figure 5. Principal features of the theory of Durgin and Li. Two angles are systematically over-perceived, angular declination (or gaze declination) and optical slant. The physical value of angular declination is represented by $\alpha$ and the corresponding perceived angle is $\alpha^{\prime}$. The physical value of optical slant is represented by $\beta$ and the corresponding perceived angle is $\beta$ '. $\mathrm{D}$ is the egocentric distance to a target on the ground plane, and D' is the corresponding perceived distance.

surface where the target is located and the surface itself. The authors have conducted various experiments in which observers consistently overestimate both types of angles. Because this overestimation is found using a variety of response methods such as verbal report and reproduction, the authors conclude that the perceived angles are larger than the corresponding physical angles. From $0^{\circ}$ to at least $45^{\circ}$, perceived angular declination is proportional to the corresponding physical values with a gain (multiplicative factor) of 1.5 (Durgin \& Li, 2011). Perceived optical slant is also over-perceived, but the degree of over-perception varies considerably with distance of the surface, the task, and other factors (Durgin \& Li, 2011; Durgin et al., 2010; Li \& Durgin, 2010, 2013).

As mentioned earlier, verbal reports of distance under full-cue viewing are proportional to target distance, with a gain of about 0.7 (e.g. Loomis \& Philbeck, 2008). To the extent that angular declination is the primary cue to distance of targets on the ground plane, the expansion of angular declination predicts this under-perception of distance, as measured by verbal report (Figure 5). Thus, the theory is consistent with the linearity of perceived egocentric distance for targets on the ground plane under full-cue viewing ( $\mathrm{Li}$ et al., 2011). How then does the theory explain the large distortions of perceived shape for target configurations on the ground plane under similar viewing conditions?

According to the theory, there are two possible contributions to this distortion of shape: the expansion of angular declination and the expansion of optical slant. The paper by Li, Sun, Strawser, Spiegel et al. (2013) focuses on the first factor in accounting for the anisotropy of large spatial extents. They have found that for visual directions increasing within horizontal planes away from the median plane, there is also an expansion of these angles, but the gain of this expansion is only 1.2 in contrast to the 1.5 gain for angular declination, a result which is more or less in line with the findings of Higashiyama (1992). This differential expansion means that depth extents on the ground plane are compressed more than width extents (Li et al., 2013). The paper by Li and Durgin (2010) focuses on the second factor, which is a more potent determinant of anisotropy. The authors begin by assuming that the effective visual aspect ratio used to derive perceived slant and perceived aspect ratio is the same as that of the perspective projection (i.e., retinal image). This allows them to predict the ratio of the perceived optical slant to the actual optical slant, which for this task comes out to be about 1.5 for near viewing distances and to increase with viewing distance. Direct estimates of the optical slant of sloped surfaces also support the hypothesis of expansion of optical slant and allow a fit of a revised model of perceived optical slant, which also is moderately successful in fitting the results of the aspect ratio task. In this case the predicted perceived optical slant is greater than the expansion of angular declination (see Figure 5). Li and Durgin (2010) report that the model is successful in fitting the data of Kudoh (2005), which are like those in the left panel of Figure 4. An improved model of perceived optical angle has been developed by Li and Durgin (2013).

The theory is consistent with both the linearity of egocentric distance perception and large distortions of shape. To reconcile these, Li and Durgin (2012, p. 373) propose that egocentric distance perception under full cues is mostly based on perceived angular declination, which, in spite of its expansion, predicts a linear distance function, whereas exocentric distance perception is more dependent on perceived optical slant. Thus, the theory is consistent with the hypothesis of Loomis et al. (1992, 1996) of a dissociation in processing for egocentric and exocentric perception discussed earlier.

Theory of Ooi and He. This theory came about as the result of the theoretical orientation of the two theorists and their development of a new method for measuring the perceived location of a target. Ooi and $\mathrm{He}$ were heavily influenced by James Gibson's emphasis on the role played by the ground plane in space perception (Gibson, 1950) and began doing experiments showing the importance of the ground surface (Sinai, Ooi, \& He, 1998). Shortly thereafter, Ooi and He, aided by graduate student $\mathrm{B}$. $\mathrm{Wu}$, extended the established blind walking method for measuring perceived distance to measure the perceived three-dimensional location of a single target (Ooi et al., 2001). In this seminar paper, the authors presented results of an experiment in which observers viewed a glowing target on the floor of an otherwise dark room. After viewing the target, observers blindly walked the approximate distance to the target and then attempted to gesture with the hand to the unseen location of the target. The indicated locations on average were in the same directions as the targets as viewed from the original location of the eyes, signifying the correct perception of angular declination. Equally as important, the indicated locations under 
these conditions of minimal distance cues were closer and higher than the targets, as judged from the origin. The authors concluded that the indicated locations were coincident with the perceived locations, as viewed from the origin. By presenting a variety of targets on the floor, the loci of perceived locations define what the authors refer to as the "intrinsic bias" of visual perception when all distance cues except angular declination are absent. This intrinsic bias (not to scale) is depicted in the uppermost panel of Figure 6.

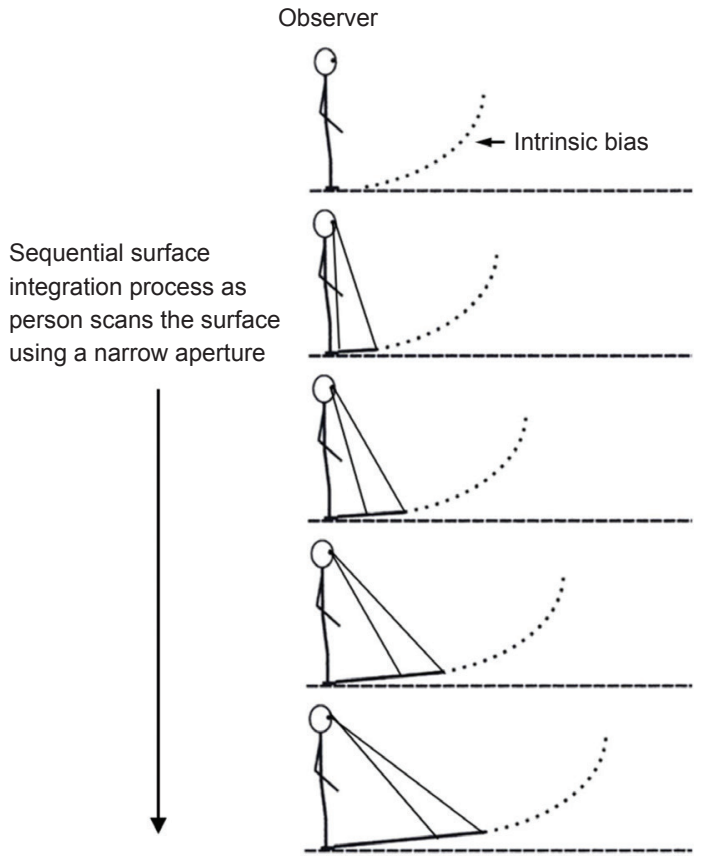

Figure 6. Side-view depiction of the "intrinsic bias" and "sequential surface integration process" of the theory of Ooi and He. Top panel: When an observer views a glowing target in an otherwise dark environment at different values of angular declination, the loci of indicated locations lie on the surface labeled "intrinsic bias"; this is the default perceptual tendency in the absence of visual cues. The remaining panels from top to bottom show that when the observer scans a visible ground plane from the feet outward (in this case through a fieldlimiting aperture), a perceptual ground surface is sequentially created, starting from the intrinsic bias and gradually extending outward based on the visible surface information.
An important question is how the visual system determines perceived locations when other distance cues are available. As is now known, the perceived locations are codetermined by the intrinsic bias and cues specifying spatial layout (Gajewski, Philbeck, Wirtz, \& Chichka, 2013; J. Wu, He, \& Ooi, 2013). In 2004, Ooi and He reported evidence of a sequential surface integration process in which a perceptual representation of the ground is sequentially built up by fixating or attending to different parts of the visible ground surface (He, J. Wu, Ooi, Yarbrough et al., 2004; B. Wu et al., 2004; J. Wu, He, \& Ooi, 2008). Figure 6 depicts the situation when the observer views the visible ground through a field-limiting aperture and sequentially scans from the feet forward. Subsequent frames depict how the surface is hypothetically built up as the gaze moves forward (He et al., 2004; B. Wu et al., 2004; J. Wu et al., 2008; J. Wu et al., 2013). Provided that there is some visual information about the ground surface, the final representation of the ground surface is less inclined than the intrinsic bias (J. Wu et al., 2013). The more visual information that is available, the more accurate is the perceptual representation. When viewing is under full cues without a field-limiting aperture, the resulting representation of the ground surface is approximately planar with a slight upward slope of several degrees (B. Wu et al., 2004; Ooi \& He, 2007). Under these conditions perceived egocentric distance is a linear or very nearly linear function of physical distance.

How then does the theory explain the large systematic misperception of shape under the same viewing conditions? The authors hypothesized (Ooi et al., 2006; p. 621) that when observers make judgments about the shape of a configuration on the ground (an exocentric task), they do not fixate or attend to the near ground surface but instead only attend to the region of the ground near the configuration. They further hypothesized that, without sequential integration of the foreground, the intrinsic bias exerts greater influence, the result being that the perceived slant of the ground is steeper (Figure 7, right panel) than when they are performing the egocentric distance task (Figure 7,
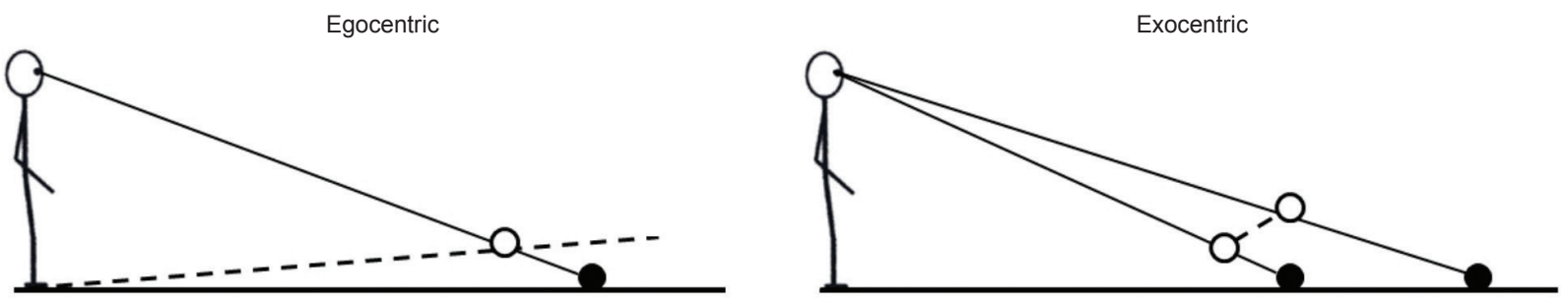

Figure 7. Side view depiction of the slope of the perceived ground surface when making egocentric and exocentric judgments according to the theory of Ooi and He. Left panel: when the observer is asked to estimate the distance to a target on a visible ground plane, the observer fixates and attends to the nearby ground surface. The sequential surface integration process, as depicted in Figure 6, gives rise to a slightly sloped perceived surface. Right panel: when the observer is judging the length of an exocentric depth interval, the observer fixates and/or attends to only the region of the ground surface near the target. Because the sequential surface integration process begins further out from the observer, the influence of the intrinsic bias is greater, resulting in a more sloped perceptual ground surface. 
left panel). Moreover, the perceived slant is predicted to increase with the distance of the targets used in the exocentric task. There is evidence for this trend in a shape judgment task under reduced-cue viewing ( $\mathrm{J}$. Wu et al., 2008, 2013) and the equal-appearing interval task (Figure 3) under full-cue viewing (Gilinsky, 1951; Ooi \& He, 2007). Because the processing of egocentric distance perception and that of exocentric distance perception are distinct, the theory, like that of $\mathrm{Li}$ and Durgin, is consistent with the hypothesis of Loomis et al. $(1992,1996)$ discussed earlier. The theory of Ooi and $\mathrm{He}$ and that of Durgin and Li directly conflict on the issue of whether angular declination is accurately perceived while also differing in terms of starting motivations and the emphasis given to different experimental methods.

\section{Perceived shape can change even with perceived locations held constant}

The three theories differ in terms of whether a unitary representation can account both for the facts of egocentric distance perception and exocentric distance perception under full-cue viewing. Loomis et al. (2002) reported assumption-free evidence for such a dissociation between egocentric and exocentric distance perception. In two experiments, observers performed two types of tasks with targets on a horizontal surface viewed with one or two eyes under good illumination. In one task they indicated the perceived locations of several targets using open-loop action measures or using verbal estimates of the target distances (assuming correct perception of direction). The other task involved judgments of L-shapes on a horizontal surface, either indicated by verbal estimates of the aspect ratio of the L-shape or by matching its depth to its width. The critical finding was that the perceived locations of targets were not measurably influenced by whether viewing was monocular or binocular, but the judged shape was significantly more veridical with binocular viewing. This finding means that the perceived shape of a configuration is not constrained by the perceived locations of its vertices, a result that is consistent with the hypothesis of a dissociation presented earlier. It is readily interpretable within the theory of Ooi and He. The effects of the monocular/binocular manipulation, however, are small in comparison with the discrepancies between the linearity of egocentric distance perception and the large distortions of shape discussed earlier.

\section{Summary}

The three theories described above came into being out of different initial concerns and were developed using different assumptions and emphasizing different experimental methods, but all three end up being relevant to the topic of this article. The theory by Foley, RibeiroFilho, and Da Silva is based on the idea that when the visual system computes the length of a visible extent, the effective visual angle is a non-linear increasing function of the actual visual angle, resulting in perceived extents that differ from the actual extents in a way that depends on how much the extent varies in depth. The model is able to account for both egocentric and exocentric extents using a unitary visual representation. The theory of Durgin and Li is based on the idea that optical slant and angular declination are both over-perceived. The theory is consistent with the linear egocentric distance perception and shape distortion, and the authors maintain that the processing of egocentric distance and that of exocentric distance are distinct, as hypothesized by Loomis et al. $(1992,1996)$. The theory of Ooi and He stems from their interest in Gibson's ground theory (1950) and their early work on the localization of single glowing targets in darkened environments, which led them to the discovery of an intrinsic bias when distance cues are absent. Subsequent work led to the sequential surface integration process, which in conjunction with different eye fixation and attention patterns for the egocentric and exocentric tasks, provides another process interpretation for the hypothesized dissociation between egocentric perception and exocentric perception. Finally, the experiments by Loomis et al. (2002) provide strong evidence of a processing dissociation between egocentric perception and exocentric perception although the effects demonstrated with the binocular/ monocular manipulation were small in comparison with the primary contrast between egocentric and exocentric perception dealt with earlier.

\section{Acknowledgment}

I thank Frank Durgin, John Foley, and Zijiang He for providing me feedback on the accuracy with which I have discussed the three theories presented here. They are not responsible for any inaccuracies that remain.

\section{References}

Baird, J. C., \& Biersdorf, W. R. (1967). Quantitative functions for size and distance judgments. Perception \& Psychophysics, 2, 161-166.

Beusmans, J. M. (1998). Optic flow and the metric of the visual ground plane. Vision Research, 38, 1153-1170.

Bian, Z. \& Andersen, G. J. (2011). Environmental surfaces and the compression of perceived visual space. Journal of Vision, 11(7), 4.

Durgin, F. H., \& Li, Z. (2011). Perceptual scale expansion: An efficient angular coding strategy for locomotor space. Attention, Perception, \& Psychophysics, 73, 1856-1870.

Durgin, F. H., Li, Z., \& Hajnal, A. (2010). Slant perception in near space is categorically biased: Evidence for a vertical tendency. Attention, Perception, \& Psychophysics, 72, 1875-1889.

Foley, J. M. (1968). Depth, size and distance in stereoscopic vision. Perception and Psychophysics, 3, 265-274.

Foley, J. M. (1972). The size-distance relation and intrinsic geometry of visual space: Implications for processing. Vision Research, 12, 323-332.

Foley, J. M. (1980). Binocular distance perception. Psychological Review, 87, 411-434.

Foley, J. M. (1991). Binocular space perception. In D. Regan (Ed.), Binocular vision (vol. 9, pp. 75-92). London: Macmillan.

Foley, J. M., Ribeiro-Filho, N. P., \& Da Silva, J. A. (2004). Visual perception of extent and the geometry of visual space. Vision Research, 44, 147-156

Gajewski, D. A., Philbeck, J. W., Wirtz, P. W., \& Chichka, D. (2013). Angular declination and the dynamic perception of egocentric distance. Journal of Experimental Psychology: Human Perception and Performance, 40, 361-377. 
Gibson J. J. (1950). The perception of the visual world. Boston: Houghton Mifflin.

Gilinsky, A. S. (1951). Perceived size and distance in visual space. Psychological Review, 58, 460-482.

Gogel, W. C. (1977). The metric of visual space. In W. Epstein (Ed.), Stability and constancy in visual perception: Mechanisms and processes, 129-181. New York: Wiley.

Gogel, W. C. (1982). Analysis of the perception of motion concomitant with a lateral motion of the head. Perception \& Psychophysics, 32, 241-250.

Gogel, W. C. (1990). A theory of phenomenal geometry and its applications. Perception \& Psychophysics, 48, 105-123.

He, Z. J., Wu, B., Ooi, T. L., Yarbrough, G., \& Wu, J. (2004). Judging egocentric distance on the ground: Occlusion and surface integration. Perception, 33, 789-806.

Higashiyama, A. (1992). Anisotropic perception of visual angle: Implications for the horizontal-vertical illusion, overconstancy of size, and the moon illusion. Perception \& Psychophysics, 51, 218-230.

Higashiyama, A., \& Ueyama, E. (1988). The perception of vertical and horizontal distances in outdoor settings. Perception \& Psychophysics, 44, 151-156.

Howard, I. P., \& Rogers, B. J. (2012). Perceiving in Depth, Volume 3, Other Mechanisms of Depth Perception. Oxford: Oxford University Press.

Kudoh, N. (2005). Dissociation between visual perception of allocentric distance and visually directed walking of its extent. Perception, 34, 1399-416.

Levin, C. A. \& Haber, R. N. (1993). Visual angle as a determinant of perceived interobject distance. Perception \& Psychophysics, 54, 250-259.

Li, Z., \& Durgin, F. H. (2009). Downhill slopes look shallower from the edge. Journal of Vision, 9 (11): 6.

Li, Z., \& Durgin, F. H. (2010). Perceived slant of binocularly viewed large-scale surfaces: A common model from explicit and implicit measures. Journal of Vision, 10(14): 13.

Li, Z., \& Durgin, F. H. (2012). A comparison of two theories of perceived distance on the ground plane: The angular expansion hypothesis and the intrinsic bias hypothesis. i-Perception, 3, 368-383.

Li, Z., \& Durgin, F. H. (2013). Depth compression based on misscaling of binocular disparity may contribute to angular expansion in perceived optical slant. Journal of Vision, 13(12):3.

Li, Z., Phillips, J., \& Durgin, F. H. (2011). The underestimation of egocentric distance: Evidence from frontal matching tasks. Attention, Perception, \& Psychophysics, 73, 2205-2217.

Li, Z., Sun, E., Strawser, C. J., Spiegel, A., Klein, B., \& Durgin, F. H. (2013). On the anisotropy of perceived ground extents and the interpretation of walked distance as a measure of perception. Journal of Experimental Psychology: Human Perception and Performance, 39, 477-493.

Loomis, J. M., Da Silva, J.A., Fujita, N., \& Fukusima, S. S. (1992). Visual space perception and visually directed action. Journal of Experimental Psychology: Human Perception and Performance, 18, 906-921.

Loomis, J. M., Da Silva, J. A., Philbeck, J. W., \& Fukusima, S. S. (1996). Visual perception of location and distance. Current Directions in Psychological Science, 5, 72-77.
Loomis, J. M. \& Philbeck, J. W. (1999). Is the anisotropy of perceived 3-D shape invariant across scale? Perception \& Psychophysics, 61, 397-402.

Loomis, J. M. \& Philbeck, J. W. (2008). Measuring spatial perception with spatial updating and action. In R. L. Klatzky, M. Behrmann, \& B. MacWhinney (Eds.), Embodiment, ego-space, and action (pp 1-43). New York: Taylor \& Francis.

Loomis, J. M., Philbeck, J. W., \& Zahorik, P. (2002). Dissociation of location and shape in visual space. Journal of Experimental Psychology: Human Perception and Performance, 28, 12021212.

Norman, J. F., Todd, J. T., Perotti, V. J., \& Tittle, J. S. (1996). The visual perception of three-dimensional length. Journal of Experimental Psychology: Human Perception and Performance, $22,173-186$

Ooi, T. L., \& He, Z. J. (2007). A distance judgment function based on space perception mechanisms: Revisiting Gilinsky's (1951) equation. Psychological Review, 114, 441-454.

Ooi, T. L., Wu, B., \& He, Z.J. (2001). Distance determined by the angular declination below the horizon. Nature, 414, 197-200.

Ooi, T. L., Wu, B., \& He, Z. J. (2006). Perceptual space in the dark affected by the intrinsic bias of the visual system. Perception, 35 , 605-624.

Proffitt, D. R., Bhalla, M., Gossweiler, R., \& Midgett, J. (1995). Perceiving geographical slant. Psychonomic Bulletin \& Review, 2, 409-428.

Sedgwick, H. A. (1986). Space perception. In K. R. Boff, L. Kaufman, \& J.P. Thomas (Eds.), Handbook of perception and human performance: Vol. 1, Sensory processes and perception (pp. 21.121.57). New York: Wiley.

Sinai, M. J., Ooi, T. L., \& He, Z. J. (1998). Terrain influences the accurate judgement of distance. Nature, 395, 497-500.

Toye, R. C. (1986). The effect of viewing position on the perceived layout of space. Perception \& Psychophysics, 40, 85-92.

Wagner, M. (1985). The metric of visual space. Perception \& Psychophysics, 38, 483-495.

Wu, B., Ooi, T. L., \& He, Z. J. (2004). Perceiving distances accurately by a directional process of integrating ground information. Nature, 428, 73-77.

Wu, J., He, Z. J., \& Ooi, T. L. (2008). Perceived relative distance on the ground affected by the selection of depth information. Perception \& Psychophysics, 70, 707-713.

Wu, J., He, Z. J. \& Ooi, T. L. (2013). The visual system's intrinsic bias influences space perception in the impoverished environment. Journal of Experimental Psychology: Human Perception and Performance. Advance online publication. doi: 10.1037/a0033034

Yang, Z., \& Purves, D. (2003). A statistical explanation of visual space. Nature Neuroscience, 6, 632-640.

Zhou, L., He, Z. J., \& Ooi, T. L. (2013). The visual system's intrinsic bias and knowledge of size mediate perceived size and location in the dark. Journal of Experimental Psychology: Learning, Memory, \& Cognition, 39, 1930-1942. 